\title{
Prevalence and Clinical Correlates of Insomnia in Depressive Disorders: The CRESCEND Study
}

\author{
Seon-Cheol Park', ${ }^{1,}$ Jae-Min Kim³, Tae-Youn Jun ${ }^{4}$, Min-Soo Lee ${ }^{5}$, \\ Jung-Bum Kim ${ }^{6}$, Seung-Hee Jeong ${ }^{7}$, and Yong Chon Park ${ }^{2,8} \bowtie$ \\ ${ }^{1}$ Department of Psychiatry, Yong-In Mental Hospital, Yongin, Republic of Korea \\ Institute of Mental Health, Hanyang University, Seoul, Republic of Korea \\ ${ }^{3}$ Department of Psychiatry, School of Medicine, Chonnam National University, Gwangju, Republic of Korea \\ ${ }^{4}$ Department of Psychiatry, College of Medicine, The Catholic University of Korea, Seoul, Republic of Korea \\ ${ }^{5}$ Department of Psychiatry, College of Medicine, Korea University, Seoul, Republic of Korea \\ ${ }^{6}$ Department of Psychiatry, Keimyung University School of Medicine, Daegu, Republic of Korea \\ ${ }^{7}$ Department of Preventive Medicine, College of Medicine, The Catholic University of Korea, Seoul, Republic of Korea \\ ${ }^{8}$ Department of Psychiatry, College of Medicine, Hanyang University, Seoul, Republic of Korea
}

Objective To investigate the prevalence, clinical manifestations, and clinical correlates of insomnia in a large cohort of Korean patients with depressive disorders.

Methods We recruited 944 patients with depressive disorders from the Clinical Research Center for Depression of South Korea (CRESCEND) study. Psychometric scales were used to assess depression (HAMD), anxiety (HAMA), psychotic symptoms (BPRS), global severity (CGI-S), and functioning (SOFAS). Insomnia levels were determined by adding the scores for all items on the HAMD insomnia subscale. The clinical characteristics of the patients with 'low insomnia' (summed score $\leq 3$ on the HAMD subscale) and 'high insomnia' (score $\geq 4$ ) were compared using statistical analyses. A logistic regression model was constructed to identify factors associated with 'high insomnia' status.

Results Symptoms of insomnia were present in $93 \%$ of patients, while simultaneous early, middle, and late insomnia affected $64.1 \%$. The high insomnia patients were characterized by significantly greater age, higher symptom levels (including core, gastrointestinal somatic and anxiety symptoms, and suicidal ideation), higher global severity and incidence of physical disorders, and greater insight. Explanatory factors of 'high insomnia' status were older age, higher gastrointestinal somatic and anxiety symptom levels, higher global severity, and greater insight.

Conclusion In clinical psychiatry, insomnia has been significantly underdiagnosed and undertreated. It affects most patients with depressive disorders, and is indicative of the global severity of depression. Active efforts to diagnose and treat insomnia in patients with depressive disorders should be strongly encouraged. Further research is needed to improve the diagnosis and treatment of insomnia in depressive patients.

Psychiatry Investig 2013;10:373-381

Key Words Insomnia, Depressive disorders, Anxiety symptoms, Gastrointestinal somatic symptoms, Global severity.

\section{INTRODUCTION}

The economic burden of depressive disorders, and particularly of major depressive disorder (MDD), in Korea, was esti-

Received: December 13, 2012 Revised: May 11, 2013

Accepted: May 14, 2013 Available online: December 16, 2013

$\triangle$ Correspondence: Yong Chon Park, MD

Department of Psychiatry, Hanyang University Guri Hospital, 153 Gyeongchun-ro, Guri 471-701, Republic of Korea

Tel: +82-31-560-2273, Fax: +82-31-554-2599, E-mail: hypyc@hanyang.ac.kr

(c) This is an Open Access article distributed under the terms of the Creative Commons Attribution Non-Commercial License (http://creativecommons.org/licenses/bync/3.0) which permits unrestricted non-commercial use, distribution, and reproduction in any medium, provided the original work is properly cited. mated to exceed 1.5 billion USD in $2004,{ }^{1}$ and depression has been identified as the most important mental disorder in the Korean population. ${ }^{2,3}$ Depression encompasses a wide range of symptoms affecting mood, cognition, and motor functions, causing patients to experience psychosocial problems and impaired quality of life. In patients with depression, insomnia is often under-detected and under-treated. ${ }^{4}$ Yet, it is estimated that around three quarters of them experience insomnia symptoms. ${ }^{5}$

The relationship between insomnia and depressive disorders is reciprocal, as they share fundamental neurobiological underpinnings. ${ }^{6}$ A growing body of evidence suggests that chron- 
ic insomnia is a strong risk factor for the development of depression $^{7}$ as well as a prognostic indicator of clinical course and response to treatment in depressive disorders. ${ }^{8-10}$ In addition, insomnia is associated with suicidal ideation and behavior in depressive patients. ${ }^{11}$ Although insomnia can manifest in a number of psychiatric illnesses, it has been shown to be a core symptom and an independent risk factor for MDD. ${ }^{12,13}$

In a recent large epidemiological study of unipolar and nonpsychotic MDD patients in the United States (the STAR*D study), $84.7 \%$ of depressive participants experienced insomnia, and insomnia was also correlated with scores reflecting more severe depression. ${ }^{4}$ In Korea, insomnia is the most common symptom for which depressive patients receive psychiatric treatment. ${ }^{14}$ Although depressive disorders have a considerable impact on the mental health of the Korean population, most research in this area has been limited to biological psychiatry. The Clinical Research Center for Depression (CRESCEND) study was the first large, prospective, observational, clinical study of a nationwide sample of Korean patients with depressive disorders that provided epidemiological data. ${ }^{3}$

Using the data collected in the CRESCEND study, our aim was to: 1) estimate the prevalence of insomnia in patients with depressive disorders; 2) identify socio-demographic and clinical characteristics, and assessment scale scores, associated with 'low insomnia' and 'high insomnia' status, respectively, in patients with depressive disorders; and 3) identify significant socio-demographic and clinical correlates of insomnia in patients with 'high insomnia' status.

\section{METHODS}

\section{Study overview}

From January 2006 to August 2008, 1183 patients with depressive disorders were enrolled in the CRESCEND study at one or other of 18 study centers across South Korea, including 16 university-affiliated hospitals and two general hospitals. The study was conducted over a period of nine years. During phase I, eligible subjects were assessed at baseline, followed by assessments at weeks $1,2,4,8,12,24$, and 52 . In phase II, the subjects were assessed annually over the course of eight years. The data management center, which monitored the collection and quality of the data, was situated in the Preventative Medicine Department of the Catholic University College of Medicine in Seoul. All socio-demographic and clinical data were collected by trained and certified clinical research coordinators supervised by clinical psychiatrists at the regional centers. All patient data were recorded using a preselected clinical report form and stored on the CRESCEND study website (www.smileagain.or.kr).

\section{Study subjects}

In order to closely reflect real clinical situations, the study had broad inclusion and minimal exclusion criteria. We enrolled psychiatric in and outpatients who were beginning new treatment for first-onset or recurrent depression. The inclusion criteria were as follows: 1) age over seven years, and 2) presence of MDD (with or without psychotic features), dysthymia, or depressive disorders not otherwise specified, diagnosed according to DSM-IV criteria ${ }^{15}$. The exclusion criteria were: 1 ) current or lifetime diagnosis of schizophrenia, other psychotic disorders, bipolar disorder, organic psychosis, or dementia, according to DSM-IV criteria; 2) presence of a medical or neurological illness severe enough to interfere with study evaluations and interviews; and/or 3) breastfeeding, pregnancy, or intention to become pregnant within nine months of enrolment. Out of the 1183 patients enrolled in the CRESCEND study, we selected the records of 944 patients aged 18-75 years with a baseline Hamilton Depression Rating Scale (HAMD) score $\geq 14$ points, for the purpose of statistical analysis.

All relevant university and/or hospital Institutional Review Boards (IRBs) approved the study protocol and consent forms. All study participants, or their authorized representatives, provided written informed consent prior to participation.

\section{Clinical presentation and levels of insomnia}

As the CRESCEND study was designed to provide epidemiological data about depression rather than sleep, it did not employ polysomnography. Instead, insomnia symptoms were evaluated using a 3-item insomnia subscale on the 17 -item Hamilton Depression Rating Scale (HAMD). ${ }^{16}$ These three items, scored on a scale of 0 -2, address inability to fall asleep (early insomnia), waking during the night (middle insomnia), and waking too early (late insomnia). The substantial agreement between scores on the HAMD insomnia subscale and sleep diary data has validated the HAMD subscale as a global measure of insomnia severity in depressive disorders. ${ }^{17}$ In large-sample-sized studies, sleep-specific evaluation methods that include polysomnography, sleep assessment scales, and sleep diaries are occasionally substituted by the insomnia items of the HAMD. Thus, the insomnia items of the HAMD were defined as the main outcome variables. ${ }^{18-21}$ The presence of insomnia was indicated by a score of $\geq 1$ on the HAMD insomnia subscale. ${ }^{18}$ Insomnia levels were determined by summing the scores for all items on the HAMD insomnia subscale A comparison of the clinical characteristics between patients with high and low levels of insomnia was needed in order to effectively determine the significant clinical correlates of insomnia. Thus, the 2 subgroups were divided according to an operational cut-off value that has been used in the previous study. According to Montgomery et al's conceptualization, ${ }^{22}$ 
high insomnia was defined as individual scores that summed to 4 or more, and low insomnia was defined as individual scores that summed to 3 or less.

\section{Assessment scales}

The socio-demographic data collected on the clinical report forms included age, sex, length of education, marital status, cohabitation status, religious affiliation, current occupation, and monthly income. Diagnostic evaluations and retrospective reporting of the histories of medical and/or psychiatric illnesses and treatments took place at baseline. Diagnoses of depressive disorders were made by the attending clinicians using DSM-IV criteria. ${ }^{15}$ The diagnoses were confirmed in week 2 in a subsample of patients, using a DSM-IV-based Structured Clinical Interview (SCID) ${ }^{23}$ Depressive disorders were categorized as follows: 1) MDD without psychotic features; 2) MDD with psychotic features; 3) dysthymia; and 4) depressive disorders not otherwise specified. The clinical data collected in the study included inpatient/outpatient status, first-time or recurrent disease, number of previous depressive episodes, age at onset, duration of illness, family history of depression, history of suicide attempts, and concurrent physical disorders. The section concerning suicide attempts had additional questions about the method and severity of each attempt. The section concerning concurrent physical disorders had questions about the presence or absence of 33 listed physical disorders, including hypertension, cancer, diabetes, angina, cerebrovascular disease, thyroid disease, osteoporosis, hyperlipidemia, Cushing's disease, uremia, gastroduodenal ulcer, and inflammatory bowel disease. As the treatment statuses and severities of the physical disorders were also questioned, patients with a concurrent medical or neurological illness that was severe enough to interfere with study participation were excluded.

The clinician-administered scales, including the 17-item Hamilton Depression Rating Scale (HAMD), ${ }^{16}$ the Hamilton Anxiety Rating Scale (HAMA), ${ }^{24}$ the Brief Psychiatric Rating Scale (BPRS), ${ }^{25}$ the Clinical Global Impression of Severity scale (CGI-S) ${ }^{26}$ and the Social and Occupational Functioning Assessment Scale (SOFAS), ${ }^{27}$ were used to evaluate the severity of the depressive symptoms, severity of the anxiety symptoms, severity of the psychotic symptoms, global severity, and levels of social and occupational functioning, respectively. All of these scales were formally translated into Korean, and they have been demonstrated to be valid and reliable in Korean populations. ${ }^{28,29}$ Higher scores on the HAMD, the HAMA, the BPRS, and the CGI-S represent more severe individual scalespecific symptoms, while lower scores on the SOFAS indicate poorer function.

The core symptoms of depression were defined using a 6-item HAMD scale developed by Evans et al., ${ }^{30}$ and the sever- ity of depression was assessed by adding the scores for these items, including depressed mood, guilt, work and activities, psychic anxiety, somatic anxiety, and general somatic symptoms. The 6-item HAMD had been initially developed to increase the signal-to-noise ratio and reduce the failure rate in efficacy trials of antidepressants. ${ }^{31}$ In the present study, the scale was used to investigate the relationship between insomnia and the 'core', 'associated' and 'co-morbid' symptoms of depressive disorders. The total scores on the HAMD and HAMA scales were calculated by adding the scores for all except the insomnia-related items, in order to control for the effects of insomnia when comparing the scores of low and high insomnia patients. Moreover, all individual items of the HAMD without the insomnia subscales were used to compare all of the multiple dimensions of the depressive symptoms between patients with high and low insomnia with the same study design as that used in a previous investigation.

\section{Statistical analysis}

Descriptive statistics were used to determine the distribution of symptoms and levels of insomnia among the patients. Accordingly, insomnia was categorized as early, middle, and late, and symptom levels ranged from 0 to 6 . Socio-demographic and clinical characteristics and the assessment scale scores were compared in the low and high insomnia groups using an independent $t$-test for continuous variables and a $\chi^{2}$ test for categorical variables. A logistic regression model was constructed to identify factors associated with 'high insomnia' status. In the model, the patients with 'high insomnia' status were defined as the dependent variable, while the low insomnia group was a reference category. Socio-demographic and clinical variables shown to be significantly different between the two groups were used as covariates. Standard methods such as goodness of fit, interaction, and colinearity were used to select and validate the final model. Significance was set at $\mathrm{p}<0.05$ (two-tailed) for all tests. All statistical analyses were performed using SPSS 18.0 for Windows (SPSS Inc., Chicago, IL, USA).

\section{RESULTS}

\section{Patient characteristics}

Table 1 and 2 summarize the socio-demographic and clinical characteristics of the 994 patients. The mean age was 56 $(\mathrm{SD}=14.6)$ years, and most of the patients were female $(74.7 \%)$, married (60.3\%), unemployed (59.2\%), with a religious affiliation (60.2\%), diagnosed with MDD without psychotic symptoms (82.9\%), and enrolled as outpatients (78.1\%). The mean score on the HAMD scale without insomnia was $17.7(\mathrm{SD}=4.3)$, followed by 18.1 ( $\mathrm{SD}=7.9)$ on the HAMA without insomnia, 
$0.4(\mathrm{SD}=1.1)$ on the BPRS, $4.8(\mathrm{SD}=1.0)$ on the CGI-S scale, and $57.2(\mathrm{SD}=10.8)$ on the SOFAS.

\section{Prevalence of insomnia}

The prevalence of insomnia according to the level of symptoms is shown in Figure 1. A score of 6 on the HAMD scale was reported in $20.9 \%$ of patients, and was the most commonly reported score on the 0-6 scale. 'High insomnia' was more frequent (59.1\%) than 'low insomnia' (40.1\%).

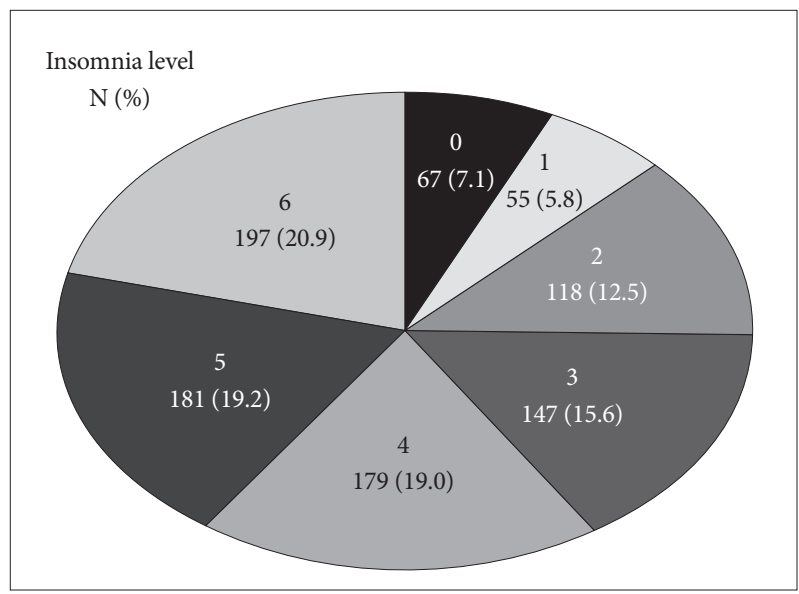

Figure 1. Insomnia levels of the patients with depressive disorders in the CRESCEND study.
The prevalence of insomnia according to its clinical presentations is shown in Figure 2. Approximately $92.9 \%$ of the 994 patients suffered from some type of insomnia. Remarkably, the simultaneous presence of early, middle, and late insomnias was the most common of all insomnia presentations (64.1\%). Overall, middle insomnia was the most frequent of the three types. However, on their own each of the three types was present in $<5 \%$ of the patients.

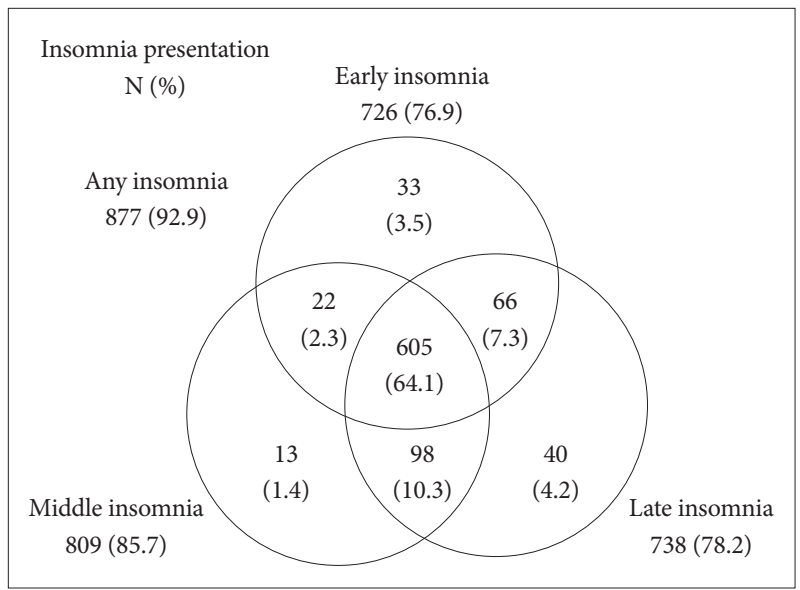

Figure 2. Insomnia presentations of the patients with depressive disorders in the CRESCEND study.

Table 1. Socio-demographic and clinical characteristics of the subjects

\begin{tabular}{|c|c|c|c|c|c|}
\hline & $\begin{array}{c}\text { Total sample } \\
\quad(\mathrm{N}=944)\end{array}$ & $\begin{array}{c}\text { Low insomnia } \\
(\mathrm{N}=387 ; 40.9 \%)\end{array}$ & $\begin{array}{l}\text { High insomnia } \\
(\mathrm{N}=557 ; 59.1 \%)\end{array}$ & $\begin{array}{c}\text { Statistical } \\
\text { coefficients }\end{array}$ & p-value \\
\hline \multicolumn{6}{|l|}{ Socio-demographic characteristics } \\
\hline Age, mean (SD) years & $50.6(14.6)$ & $48.9(15.2)$ & $51.8(14.1)$ & $\mathrm{t}=-2.991$ & 0.003 \\
\hline Women, N (\%) & $705(74.7)$ & $291(75.2)$ & $414(74.5)$ & $\chi^{2}=0.065$ & 0.799 \\
\hline Married, N (\%) & $570(60.3)$ & $223(62.9)$ & $347(67.8)$ & $\chi^{2}=5.400$ & 0.369 \\
\hline Religious affiliation, N (\%) & $569(60.2)$ & $216(60.0)$ & $333(59.8)$ & $\chi^{2}=3.899$ & 0.564 \\
\hline Education, mean $(\mathrm{SD})$ years & $10.5(4.4)$ & $10.6(4.4)$ & $10.5(4.4)$ & $\mathrm{t}=0.479$ & 0.632 \\
\hline Unemployed, N (\%) & $559(59.2)$ & $223(56.8)$ & $336(65.6)$ & $\chi^{2}=4.868$ & 0.978 \\
\hline Monthly income <2000 USD, N (\%) & $447(47.4)$ & $175(65.2)$ & $272(48.8)$ & $\chi^{2}=2.317$ & 0.888 \\
\hline \multicolumn{6}{|l|}{ Clinical characteristics } \\
\hline Diagnosis, N (\%) & & & & $\chi^{2}=9.897$ & 0.019 \\
\hline Major depressive disorder without psychotic features & $783(82.9)$ & $320(82.7)$ & $463(83.1)$ & & \\
\hline Major depressive disorder with psychotic features & $21(2.2)$ & $7(1.8)$ & $14(2.5)$ & & \\
\hline Dysthymia & $26(2.8)$ & $18(4.7)$ & $8(1.4)$ & & \\
\hline Depressive disorder not otherwise specified & $114(12.1)$ & $42(10.9)$ & $72(12.9)$ & & \\
\hline Previous history of depression, N (\%) & $395(41.8)$ & $175(45.9)$ & $220(39.6)$ & $\chi^{2}=3.667$ & 0.056 \\
\hline Outpatient enrollment, N (\%) & $737(78.1)$ & $314(42.6)$ & $423(57.4)$ & $\chi^{2}=3.599$ & 0.058 \\
\hline Current suicidal intent, N (\%) & $502(53.2)$ & $210(55.7)$ & $292(53.6)$ & $\chi^{2}=3.288$ & 0.193 \\
\hline Previous suicidal attempt, N (\%) & $216(22.9)$ & $80(20.7)$ & $136(24.4)$ & $\chi^{2}=1.815$ & 0.182 \\
\hline Comorbid physical disorder, N (\%) & $313(33.2)$ & $107(27.6)$ & $206(37.0)$ & $\chi^{2}=8.979$ & 0.003 \\
\hline Family history of depression, N (\%) & $395(41.8)$ & $60(15.5)$ & $76(13.6)$ & $\chi^{2}=0.640$ & 0.424 \\
\hline
\end{tabular}




\section{Comparison of socio-demographic and clinical characteristics of the low and high insomnia patients}

The socio-demographic and clinical characteristics of the low and high insomnia patients are compared in Table 1. The high insomnia patients were significantly older $(\mathrm{t}=-2.991$, $\mathrm{p}=0.003)$, were more frequently diagnosed with MDD $\left(\chi^{2}=9.897, \mathrm{p}=0.019\right)$, and more frequently had physical disorders $\left(\chi^{2}=8.979, \mathrm{p}=0.003\right)$. The differences between the 2 groups with respect to gender $\left(\chi^{2}=0.065, p=0.799\right)$, current suicidal intent $\left(\chi^{2}=3.288, \mathrm{p}=0.193\right)$, previous suicidal attempts $\left(\chi^{2}=1.815, p=0.182\right)$, outpatient enrollment $\left(\chi^{2}=3.599\right.$, $\mathrm{p}=0.058)$, previous history of depression $\left(\chi^{2}=3.667, \mathrm{p}=0.056\right)$, or family history of depression $\left(\chi^{2}=0.640, p=0.424\right)$ were not significant.

\section{Comparison of assessment scale scores of the low and high insomnia patients}

A comparison of the assessment scale scores of the low and high insomnia patients is presented in Table 2. The high insomnia group had a significantly higher total score on the HAMD scale without insomnia items $(\mathrm{t}=-3.053, \mathrm{p}<0.0001)$, which included higher individual scores for suicidal ideation $(\mathrm{t}=-2.068, \mathrm{p}=0.039)$, gastrointestinal somatic symptoms $(\mathrm{t}=$ $-3.895, \mathrm{p}<0.0001)$, and core symptoms $(\mathrm{t}=-3.024, \mathrm{p}=0.003)$. Conversely, the high-insomnia group had a significantly lower score for insight $(\mathrm{t}=3.178, \mathrm{p}=0.002)$. The total scores on the

Table 2. Patients' mean (SD) scores on the assessment scales

\begin{tabular}{|c|c|c|c|c|c|}
\hline & $\begin{array}{c}\text { Total sample } \\
(\mathrm{N}=944)\end{array}$ & $\begin{array}{l}\text { Low insomnia } \\
(\mathrm{N}=387 ; 40.9 \%)\end{array}$ & $\begin{array}{l}\text { High insomnia } \\
(\mathrm{N}=557 ; 59.1 \%)\end{array}$ & Statistical coefficients & p-value \\
\hline HAMD (without insomnia items) & $17.7(4.3)$ & $17.2(3.6)$ & $18.1(4.8)$ & $t=-3.053$ & $<0.0001$ \\
\hline Depressed mood & $2.3(0.9)$ & $2.3(0.9)$ & $2.3(0.9)$ & $t=-1.505$ & 0.133 \\
\hline Feelings of guilt & $1.1(0.9)$ & $1.1(0.8)$ & $1.1(0.9)$ & $\mathrm{t}=0.645$ & 0.519 \\
\hline Suicidal ideation & $1.3(1.0)$ & $1.2(1.0)$ & $1.3(1.1)$ & $t=-2.069$ & 0.039 \\
\hline Work and activities & $2.1(0.8)$ & $2.0(0.7)$ & $2.2(0.8)$ & $\mathrm{t}=-2.970$ & 0.003 \\
\hline Psychomotor retardation & $0.8(0.7)$ & $0.8(0.7)$ & $0.8(0.7)$ & $\mathrm{t}=-0.423$ & 0.673 \\
\hline Psychomotor agitation & $1.3(0.8)$ & $1.3(0.8)$ & $1.3(0.8)$ & $\mathrm{t}=0.225$ & 0.822 \\
\hline Psychological anxiety & $2.0(0.9)$ & $2.0(0.8)$ & $2.1(0.9)$ & $t=-1.553$ & 0.121 \\
\hline Somatic anxiety & $1.4(0.8)$ & $1.4(0.8)$ & $1.5(0.8)$ & $t=-1.665$ & 0.096 \\
\hline Gastrointestinal somatic symptoms & $0.9(0.6)$ & $0.8(0.6)$ & $0.9(0.5)$ & $\mathrm{t}=-3.895$ & $<0.0001$ \\
\hline General somatic symptoms & $1.1(0.5)$ & $1.1(0.5)$ & $1.1(0.6)$ & $\mathrm{t}=-1.540$ & 0.124 \\
\hline Genital symptoms & $1.0(0.8)$ & $1.0(0.8)$ & $1.1(0.8)$ & $\mathrm{t}=-1.403$ & 0.161 \\
\hline Hypochondria & $1.2(1.0)$ & $1.2(1.0)$ & $1.2(1.0)$ & $\mathrm{t}=0.057$ & 0.954 \\
\hline Weight loss & $0.6(0.8)$ & $0.6(0.8)$ & $0.7(0.9)$ & $\mathrm{t}=-1.448$ & 0.148 \\
\hline Insight & $0.5(0.6)$ & $0.5(0.8)$ & $0.4(0.9)$ & $\mathrm{t}=3.178$ & 0.002 \\
\hline Core symptoms scale (Evans et al.) & $10.2(2.7)$ & $9.9(2.3)$ & $10.4(2.9)$ & $\mathrm{t}=-3.024$ & 0.003 \\
\hline HAMA (without insomnia item) & $18.1(7.9)$ & $16.8(7.1)$ & $19.0(8.2)$ & $\mathrm{t}=-4.489$ & $<0.0001$ \\
\hline BPRS & $33.6(9.2)$ & $32.6(9.2)$ & $34.2(9.2)$ & $\mathrm{t}=-1.257$ & 0.210 \\
\hline CGI-S scale & $4.8(1.0)$ & $4.5(0.9)$ & $5.0(1.0)$ & $t=-6.986$ & $<0.0001$ \\
\hline SOFAS & $57.2(10.8)$ & $58.0(10.2)$ & $56.6(11.2)$ & $\mathrm{t}=1.930$ & 0.054 \\
\hline
\end{tabular}

HAMD: Hamilton Depression Rating Scale, HAMA: Hamilton Anxiety Rating Scale, BPRS: Brief Psychiatric Rating Scale, CGI-S: Clinical Global Impression of Severity, SOFAS: Social and Occupational Functional Assessment Scale

Table 3. Logistic regression model showing clinical correlates of 'high insomnia' status

\begin{tabular}{lcccccc}
\hline & $\mathrm{B}$ & Standard error & Wald & p-value & Odds ratio & 95\% CI \\
\hline Age, years & 0.014 & 0.005 & 7.824 & 0.005 & 1.014 & $1.004-1.023$ \\
Gastrointestinal somatic symptoms (HAMD) & 0.293 & 0.128 & 5.200 & 0.023 & 1.340 & $1.042-1.724$ \\
Insight (HAMD) & -0.357 & 0.124 & 8.220 & 0.004 & 0.700 & $0.549-0.893$ \\
HAMA (without insomnia items) & 0.041 & 0.009 & 19.323 & $<0.0001$ & 1.041 & $1.023-1.061$ \\
CGI-S scale & 0.425 & 0.076 & 30.952 & $<0.0001$ & 1.530 & $1.317-1.777$ \\
\hline
\end{tabular}

HAMD: Hamilton Depression Rating Scale, HAMA: Hamilton Anxiety Rating Scale, CGI-S: Clinical Global Impression of Severity 
HAMA scale without insomnia items $(\mathrm{t}=-4.489, \mathrm{p}<0.0001)$ and the CGI-S scale ( $\mathrm{t}=-6.986, \mathrm{p}<0.0001)$ were higher in the high insomnia patients. However, there were no significant differences between the 2 groups with respect to the scores on the BPRS $(\mathrm{t}=-1.257, \mathrm{p}=0.210)$ and SOFAS $(\mathrm{t}=1.930, \mathrm{p}=0.054)$.

\section{Factors associated with 'high insomnia' status in logistic regression}

Table 3 presents the results of a logistic regression analysis to identify factors associated with 'high insomnia' status. The Hosmer-Lemeshow goodness-of-fit test confirmed the accuracy of the logistic model $\left(\chi^{2}=4.132, \mathrm{df}=8\right.$, and $\left.\mathrm{p}=0.845\right)$. The initial covariates selected for the logistic model included age, dichotomized diagnosis, presence of co-morbid physical disorders, individual scores (including suicidal ideations, gastrointestinal somatic symptoms, insight, and core symptoms), overall score on the HAMA scale without insomnia items, and overall score on the CGI-S scale. Because the individual scores on the 17-item HAMD scale without insomnia items were used as covariates, the total score was not.

As a result of forward selection to avoid colinearity, the final model was found to include age $(\mathrm{OR}=1.014,95 \% \mathrm{CI}=1.004$ $1.023, \mathrm{p}=0.005)$, individual scores for gastrointestinal somatic symptoms $(\mathrm{OR}=1.340,95 \% \mathrm{CI}=1.042-1.724, \mathrm{p}=0.023)$ and insight $(\mathrm{OR}=0.700,95 \% \mathrm{CI}=0.549-0.893, \mathrm{p}=0.004)$, overall score on the HAMA scale without insomnia items $(\mathrm{OR}=1.041,95 \%$ $\mathrm{CI}=1.023-1.061, \mathrm{p}<0.0001)$, and overall score on the CGI-S scale $(\mathrm{OR}=1.530,95 \% \mathrm{CI}=1.317-1.777, \mathrm{p}<0.0001)$. By contrast, dichotomized diagnosis, co-morbid physical disorders, suicidal ideation, and core symptoms were not significantly related to 'high insomnia' status.

\section{DISCUSSION}

Our analysis revealed that most patients with depressive disorders in the CRESCEND study suffered from some form of insomnia, with simultaneous early, middle, and late insomnia as the most common presentation. This evidence lends support to the finding that insomnia is the most common complaint associated with depression, being reported by more than $90 \%$ of depressive patients.

Figure 3 compares our findings with those of the STAR*D study by Sunderajan et al. ${ }^{4}$ showing the differences in insomnia prevalence according to clinical presentations. The overall prevalence of insomnia was similar in the two studies, in spite of several differences in study design. For example, due to different inclusion/exclusion criteria, the mean age $( \pm S D)$ in the CRESCEND study (50.6 \pm 14.6 years) was higher than in STAR*D (37.3 \pm 13.6 years among patients without insomnia, and $41.1 \pm 13.1$ years among insomniacs). Furthermore, unlike $\mathrm{STAR}^{*} \mathrm{D}$, the CRESCEND study recruited inpatients and patients with psychotic MDD. Any discrepancies in insomnia prevalence could also be explained by differences in the psychometric instruments and assessment schedules of the two studies. While CRESCEND used the HAMD scale at baseline, the STAR*D study used the 30-item Inventory of Depressive Symptomatology, Clinician-Rated (IDS-C30) scale, ${ }^{33}$ within 72 hours of baseline. A comparison of the prevalence of insomnia according to its clinical presentations is also shown in Figure 3. The prevalence of simultaneous early, middle and late insomnia symptoms was much higher in the CRESCEND than in the STAR*D study (64.1\% vs. $27.1 \%)$. Conversely, the prevalence of middle insomnia alone was much lower in the CRESCEND study (1.4\% vs. $13.5 \%$ ), as was the prevalence of simultaneous early and middle insomnia (2.3\% vs. $19.8 \%)$. It may be that the older age and more severe depression levels of the CRESCEND study patients led to more frequent earlymorning insomnia with inability to return to sleep, and more abnormal sleep patterns. It is worth noting, however, that each item on the HAMD insomnia subscale had three possible scores $(0,1$, and 2$)$, while those on the IDS-C30 scale had four $(0,1,2$, and 3$)$. Thus, the specificity of the HAMD scale might be lower than that of the IDS-C30.

The high insomnia patients in our study were significantly older than those with low insomnia scores. This agrees with previous reports, which showed that the prevalence and sever-

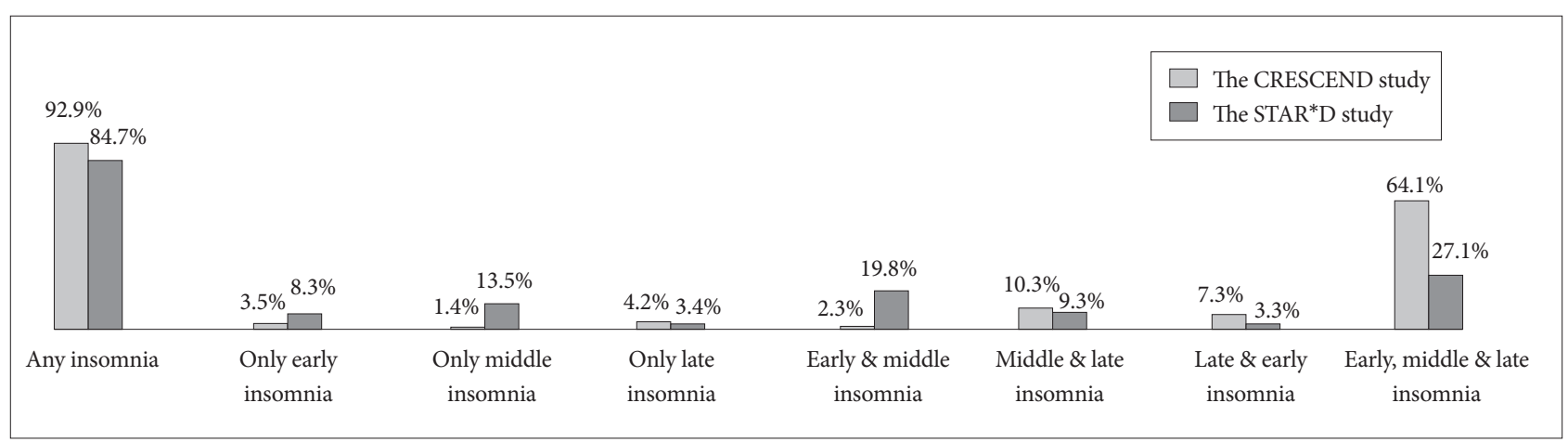

Figure 3. Comparison of the frequencies of insomnia and insomnia presentations in the CRESCEND and STAR*D surveys. 
ity of insomnia in depressive patients increased with age. ${ }^{34}$ Several studies have also associated insomnia with a higher illness burden in depressive disorders, and identified it as a risk factor for severe depression, linked to a higher probability of recurrence or relapse. ${ }^{6,35-37}$ In line with these findings, our patients with high insomnia scores had a significantly higher frequency of MDD diagnoses, and higher total scores on the HAMD scale (without insomnia items) and the CGI-S scale.

Previous reports have identified a close and reciprocal relationship between insomnia, depression, and physical disorders. ${ }^{38}$ In line with these findings, physical co-morbidities were significantly more common in the high insomnia patients in our study. There is considerable evidence that insomnia in depressive patients is associated with the severity and number of physical disorders. A cross-sectional primary care study in New Zealand showed that half of the 1517 insomniac patients had depression, and 43\% suffered from physical illnesses. ${ }^{39}$ A longitudinal community study of late life in 909 Koreans revealed that baseline insomnia was associated with incident depression and an increase in reported physical disorders, while the number of physical disorders at baseline was associated with the prevalence, incidence and persistence of insomnia. ${ }^{40}$

In terms of their performance on the HAMD scale, the high insomnia patients had significantly higher scores relating to the core symptoms identified by Evans et al. ${ }^{30}$ (including depressed mood, guilt, work and activities, psychic anxiety, somatic anxiety and general somatic symptoms) than the low insomnia group. Conversely, their score for insight was significantly lower than that of the low insomniacs. These scores concur with previous research, which suggests that insomnia is closely related to the core symptoms of depression. Taylor et al. ${ }^{41}$ showed that the number of nocturnal awakenings was associated with depression severity, and the frequency of insomnia was associated with both depression and anxiety. Another study found evidence of a relationship between severe insomnia in depressive patients, longer duration of the current depressive episode, and higher HAMD scores. ${ }^{42}$ It has also been reported that sleep disturbance and core mood symptoms improve at similar rates in response to pharmacological treatment of late-life depression. ${ }^{35}$ On the whole, the evidence suggests a link between insomnia and the core symptoms of depression, and between insomnia and the development of insight. However, it has been suggested that the association between insomnia and depression is not limited to the core symptoms. ${ }^{43}$ The high insomnia group in our study also had higher scores for gastrointestinal somatic symptoms on the HAMD scale and on the HAMA scale without insomnia than the low insomnia patients.

Although there were no significant differences in the scores for current suicidal intent and previous suicidal attempts between the low and high insomnia patients, the suicidal ide- ation score on the HAMD scale was higher in those with 'high insomnia' status. Chellappa and Araújo ${ }^{44}$ demonstrated that, although depressive patients with insomnia had significantly higher scores on the Beck Scale for Suicidal Ideation (SSI), only insomnia remained significantly associated with suicidal ideation in a multivariate analysis. The fact that a suicide-specific scale was not used in our study might explain this difference in results. Overall, the evidence suggests that insomnia in depressive patients is associated with suicidal ideation, rather than with current suicidal intent or past suicidal attempts.

Our final logistic regression model revealed that older age, less-impaired insight, and higher levels of gastrointestinal symptoms, anxiety symptoms and global severity were independent explanatory factors of 'high insomnia' status. Thus, insomnia symptoms in patients with depressive disorders may be an indicator of the global severity of depression. At the same time, the model suggested that insomnia symptoms were more closely related to the associated symptoms of depression (e.g., gastrointestinal somatic and anxiety symptoms) than with its core symptoms (e.g., mood symptoms), which contradicts the view that insomnia is an essential constitutive symptom of depressive disorders. ${ }^{12}$ While the nature of this relationship may require further investigation, our study provides more evidence that insomnia is very closely related to depressive disorders.

Our study has several limitations. Firstly, we did not employ specific methods for measuring insomnia such as polysomnography, the sleep assessment scale, or sleep diaries. As mentioned before, measuring insomnia with a tool that has low specificity could result in overestimation of its prevalence. Secondly, we did not investigate the possible impact of co-morbid psychiatric disorders on insomnia symptoms, and hence this could not be ruled out. Thirdly, the clinical significance of the statistical differences between the high and low insomnia groups was small. While this finding could be an effect of the large sample size, or the broad inclusion criteria, it may have important implications for clinical practice. The broad inclusion criteria also contributed to the considerable heterogeneity of the patients, suggesting that confounding factors may have influenced some of our findings Finally, we did not perform further statistical analyses, such as Bonferroni corrections, in the comparisons of the clinical characteristics between the patients with high and low insomnia. Thus, this was a limitation in the interpretation of the clinical significance of several statistical values in this study.

\section{Conclusions}

Insomnia affects the vast majority of patients with depressive disorders in Korea, and the simultaneous presence of early, middle, and late insomnia symptoms is very common. Our 
findings support the thesis that some insomnia cases remain under-diagnosed and under-treated in psychiatric clinical practice. Although the relationship between insomnia and depression is not yet fully understood, insomnia could be one of most clinically important symptoms in depressive disorders. Since it is closely related to global severity and less- impaired insight in depressive patients, insomnia could be used as an indicator of the clinical severity of depression. Active efforts to diagnose and treat insomnia should be strongly encouraged, to improve the management of depressive patients. However, further research is needed to optimize methods of detection and treatment of insomnia in depressive patients.

\section{Acknowledgments}

This study was supported by a grant of the Korean Health 21 Care Technology R \& D Project, Ministry of Health and Welfare, Republic of Korea (A102065). The Ministry of Health and Welfare had no further role in study design; in the collection, analysis and interpretation of data; in the writing of the report; or in the decision to submit the paper for publication.

\section{REFERENCES}

1. Jung SH. The Socioeconomic Burden of Suicide and Depression in Korea. Seoul, Korea: National Center for Mental Health Research and Education; 2005.

2. Jang SH, Park YN, Jae YM, Jun TY, Lee MS, Kim JM, et al. The symptom frequency characteristics of the Hamilton Depression Rating Scale and possible symptom clusters of depressive disorders in Korea: The CRESCEND Study. Psychiatry Investig 2011;8:312-319.

3. Kim TS, Jeong SH, Kim JB, Lee MS, Kim JM, Yim HW, et al. The clinical research center for depression study: baseline characteristics of a Korean long-term hospital-based observational collaborative prospective cohort study. Psychiatry Investig 2011;8:1-8.

4. Sunderajan P, Gaynes BN, Wisniewski SR, Miyahara S, Fava M, Akingbala $\mathrm{F}$, et al. Insomnia in patients with depression: a $\mathrm{STAR}^{\star} \mathrm{D}$ report. CNS Spectr 2010;15:394-404.

5. Nutt D, Wilson S, Paterson L. Sleep disorders as core symptoms of depression. Dialogues Clin Neurosci 2008;10:329-336.

6. Lustberg L, Reynolds CF. Depression and insomnia: question of cause and effect. Sleep Med Rev 2000;4:253-262.

7. Ohayon MM, Roth T. Place of chronic insomnia in the course of depressive and anxiety disorders. J Psychiatr Res 2003;37:9-15.

8. Buysse DJ, Reynolds CF 3rd, Hoch CC, Houck PR, Kupfer DJ, Mazumdar S, et al. Longitudinal effects of nortriptyline on EEG sleep and the likelihood of recurrence in elderly depressed patients. Neuropsychopharmacology 1996;12:243-252.

9. Buysse DJ, Tu XM, Cherry CR, Begley AE, Kowalski J, Kupfer DJ, et al. Pretreatment REM sleep and subjective quality distinguish depressed psychotherapy remitters and nonremitters. Biol Psychiatry 1999;45: 205-213.

10. Thase ME. Antidepressant treatment of the depressed patients with insomnia. J Clin Psychiatry 1999;60(Suppl 17):28-31.

11. McCall WV, Blocker JN, D’Agostino R Jr, Kimball J, Boggs N, Lasater $\mathrm{B}$, et al. Insomnia severity is an indicator of suicidal ideation during a depression clinical trial. Sleep Med 2010;11:822-827.

12. Zimmerman M, McGlinchery JB, Young D, Chelminski I. Diagnosing major depressive disorder I: A psychometric evaluation of the DSMIV symptom criteria. J Nerv Ment Dis 2006;194:158-163.

13. Mendlewicz J. Sleep disturbances: core symptoms of major depressive disorder rather than associated or comorbid disorders. World J Biol Psychiatry 2009;10:269-275.
14. Han CS. Current State of Depression Treatment in Korea. Seoul, Korea: Proceedings of Annual Symposium of the Center for Depression, Korea University Anam Hospital, Feb 23, 2006.

15. American Psychiatric Association. Diagnostic and Statistical Manual of Mental Disorders, 4th Edition. Washington DC: American Psychiatric Association; 1994.

16. Hamilton MA. A rating scale for depression. J Neurol Neurosurg Psychiatry 1960;23:56-62.

17. Manber R, Blasey C, Arnow, B, Markowitz JC, Thase ME, Rush AJ, et al. Assessing insomnia severity in depression: comparison of depression and sleep diaries. J Psychiatr Res 2005;39:481-488.

18. Na HR, Kang EH, Yu BH, Woo JM, Kim YR, Lee SH, et al. Relationship between personality and insomnia in panic disorder patients. Psychiatry Investig 2011;8:102-106.

19. Troxel WM, Kupfer DJ, Reynolds CF 3rd, Frank E, Thase ME, Miewald $\mathrm{JM}$, et al. Insomnia and objectively measured sleep disturbances predict treatment outcome in depressed patients treated with psychotherapy or psychotherapy-pharmacotherapy combinations. J Clin Psychiatry 2012;73:478-485.

20. Carli V, Roy A, Bevilacqua L, Maggi S, Cesaro C, Sarchiapone M. Insomnia and suicidal behaviour in prisoners. Psychiatry Res 2011;185: 141-144.

21. Yang H, Sinicropi-Yao L, Chuzi S, Youn SJ, Clain A, Baer L, et al. Residual sleep disturbance and risk of relapse during the continuation/ maintenance phase treatment of major depressive disorder with the selective serotonin reuptake inhibitor fluoxetine. Ann Gen Psychiatry 2010;9:10

22. Montgomery SA, Herman BK, Schweizer E, Mandel FS. The efficacy of pregabalin and benzodiazepines in generalized anxiety disorder prescribing with high levels of insomnia. Int Clin Psychopharmacol 2009;24:214-222.

23. First MB, Spitzer RL, Gibbon M, Wiliams JB. Structured Clinical Interview for DSM-IV Axis I Disorders-Patient Edition. New York: Biometrics Research Department, New York State Psychiatric Institute; 1995.

24. Hamilton MA. The assessment of anxiety state by rating. Br J Med Psychol 1959;32:50-55.

25. Overall JE, Gorham DR. The brief psychiatric rating scale. Psychol Rep 1962;10:779-812.

26. Guy W. ECDEU Assessment Manual for Psychopharmacology. US Department of Health, Education, and Welfare publication. Washington DC: National Institute of Mental Health; 1976.

27. Goldman HH, Skodol AE, Lave TR. Revising axis V for DSM-IV: a review of measures of social functioning. Am J Psychiatry 1992;149: 1148-1156.

28. Yi JS, Bae SO, Ahn YM, Park DB, Noh KS, Shin HK, et al. Validity and reliability of the Korean version of the Hamilton Depression Rating Scale (K-HDRS). J Korean Neuropsychiatr Assoc 2005;44:456-465.

29. Lee JY, Cho MJ, Kwon JS. Global assessment of functioning scale and social and occupational functioning scale. Korean J Psychopharmacol 2006;17:122-127.

30. Evans KR, Sills T, DeBrota D, Gelwicks S, Engelhardt N, Santor D. An item response analysis of the Hamilton Depression Rating Scale using shared data from two pharmaceutical companies. J Psychiatr Res 2004; 38:275-284.

31. Kennedy SH. Core symptoms of major depressive disorder: relevance to diagnosis and treatment. Dialogues Clin Neurosci 2008;10:271-277.

32. Sung G, Kim BN, Lee EH, Yu BH, Hong KS, Kim JH. Underestimating the severity of bipolar depression: a comparison of the Hamilton depression rating scale items. J Affect Disord 2012;136:425-429.

33. Trivedi MH, Rush AJ, Ibrahim HM, Carmody TJ, Biggs MM, Suppes T, et al. The Inventory of Depressive Symptomatology, Clinician Rating (IDS-C) and Self-Report (IDS-SR), and the Quick Inventory of Depressive Symptomatology, Clinician Rating (QIDS-C) and Self-Report (QIDS-SR) in public sector patients with mood disorders: a psy- 
chometric evaluation. Psychol Med 2004;34:73-82.

34. Stewart R, Besset A, Bebbington P, Brugha T, Lindesay J, Jenkins R, et al. Insomnia comorbidity and impact and hypnotic use by age group in a national survey population aged 16 to 74 years. Sleep 2006;29: 1391-1397.

35. Dombrovski AY, Blaskesley-Ball RE, Mulsant BH, Mazumdar S, Houck PR, Szanto K, et al. Speed of improvement in sleep disturbance and anxiety compared with core mood symptoms during acute treatment of depression in old age. Am J Geriatr Psychiatry 2006;14:550554.

36. Baglioni C, Battagliese G, Feige B, Spiegelhalder K, Nissen C, Voderholzer $\mathrm{U}$, et al. Insomnia as a predictor of depression: a meta-analytic evaluation of longitudinal epidemiological studies. J Affect Disord 2011;135:10-19.

37. Szklo-Coxe M, Young T, Peppard PE, Finn LA, Benca RM. Prospective associations of insomnia markers and symptoms with depression. Am J Epidemiol 2010;171:709-720

38. Langsand LE, Vatten LJ, Platou C, Janszky I. Insomnia and the risk of acute myocardial infarction: a population study. Circulation 2011;124: 2073-2781.

39. Arroll B, Fernando A 3rd, Falloon K, Goodyear-Smith F, Samaranayake C, Warman G. Prevalence of causes of insomnia in primary care: a cross-sectional study. Br J Gen Pract 2012;62:e99-e103.

40. Kim JM, Stewart R, Kim SW, Yang SJ, Shin IS, Yoon JS. Insomnia, depression, and physical disorders in late life: a 2-year longitudinal community study in Koreans. Sleep 2009;32:1221-1228.

41. Taylor DJ, Lichstein KL, Durrence HH, Riedel BW, Bush AJ. Epidemiology of insomnia, depression, and anxiety. Sleep 2005;28:1457-1464.

42. O’Brien EM, Chelminski I, Young D, Dalrymple K, Hrabosky J, Zimmerman M. Severe insomnia is associated with more severe presentation and greater functional deficits in depression. J Psychiatr Res 2011; 45:1101-1105.

43. Nekelmann D, Mykletun A, Dahl AA. Chronic insomnia as a risk factor for developing anxiety and depression. Sleep 2007;30:873-880.

44. Chellappa SL, Araújo JF. Sleep disorders and suicidal ideation in patients with depressive disordr. Psychiatr Res 2007;153:131-136. 